\title{
US nativity and dietary acculturation impact the gut microbiome in a diverse US population
}

\author{
Brandilyn A. Peters ${ }^{1,4} \cdot$ Stella S. Yi ${ }^{1} \cdot$ Jeannette M. Beasley ${ }^{2} \cdot$ Emilia N. Cobbs $^{1} \cdot$ Hee Sun Choi ${ }^{1} \cdot$ Dia B. Beggs $^{1}$. \\ Richard B. Hayes ${ }^{1,3} \cdot$ Jiyoung Ahn ${ }^{1,3}$
}

Received: 20 September 2019 / Revised: 18 February 2020 / Accepted: 28 February 2020 / Published online: 24 March 2020

(c) The Author(s), under exclusive licence to International Society for Microbial Ecology 2020

\begin{abstract}
Little is known regarding the impact of immigrant acculturation on the gut microbiome. We characterized differences in the gut microbiome between racially/ethnically diverse US immigrant and US-born groups, and determined the impact of dietary acculturation on the microbiome. Stool samples were collected from 863 US residents, including US-born (315 White, 93 Black, 40 Hispanic) and foreign-born (105 Hispanic, 264 Korean) groups. We determined dietary acculturation from dissimilarities based on food frequency questionnaires, and used 16S rRNA gene sequencing to characterize the microbiome. Gut microbiome composition differed across study groups, with the largest difference between foreign-born Koreans and US-born Whites, and significant differences also observed between foreign-born and US-born Hispanics. Differences in suboperational taxonomic unit (s-OTU) abundance between foreign-born and US-born groups tended to be distinct from differences between US-born groups. Bacteroides plebeius, a seaweed-degrading bacterium, was strongly enriched in foreign-born Koreans, while Prevotella copri and Bifidobacterium adolescentis were strongly enriched in foreign-born Koreans and Hispanics, compared with US-born Whites. Dietary acculturation in foreign-born participants was associated with specific s-OTUs, resembling abundance in US-born Whites; e.g., a Bacteroides plebeius s-OTU was depleted in highly diet-acculturated Koreans. In summary, we observed that US nativity is a determinant of the gut microbiome in a US resident population. Dietary acculturation may result in loss of native species in immigrants, though further research is necessary to explore whether acculturation-related microbiome alterations have consequences for immigrant health.
\end{abstract}

\section{Introduction}

The United States is home to the largest immigrant population in the world [1]. New immigrants to the United States tend to be healthy [2], but greater length of residence in the

Supplementary information The online version of this article (https:// doi.org/10.1038/s41396-020-0630-6) contains supplementary material, which is available to authorized users.

$\triangle$ Jiyoung Ahn

Jiyoung.Ahn@nyumc.org

1 Department of Population Health, NYU School of Medicine, New York, NY, USA

2 Department of Medicine, NYU School of Medicine, New York, NY, USA

3 NYU Perlmutter Cancer Center, New York, NY, USA

4 Present address: Department of Epidemiology and Population Health, Albert Einstein College of Medicine, Bronx, NY, USA
United States is associated with increased prevalence of obesity, diabetes, and cardiovascular disease [3-6]. Immigrants to the US experience acculturation to varying degrees; that is, the changing of lifestyle and values in response to contact with a different culture and society [7]. It has long been suspected that the acculturative process is involved in the change of immigrant disease risks toward those experienced by the US-born population.

Microbiome differences are well known between population groups defined by geographic origin and dietary and lifestyle habits [8-10]. A recent report indicates that Asian immigrants experience a "westernization" of the gut microbiome [11]; however, a large gap remains in knowledge regarding the impact of immigration and acculturation on the human gut microbiome in different racial and ethnic groups, and the specific role that dietary acculturation plays in microbiome "westernization".

In a cohort of US-born Whites, Blacks, and Hispanics and foreign-born Hispanics and Koreans, we characterized differences in the gut microbiome between racially/ 
ethnically diverse US immigrant and US-born groups, and examined the impact of dietary acculturation on the gut microbiome in the immigrant participants.

\section{Materials and methods}

\section{Parent cohort}

The Food and Microbiome Longitudinal Investigation (FAMiLI) is an ongoing prospective cohort study utilizing clinic, community, and web-based recruitment in New York City and surrounding geographic areas to achieve a racially and ethnically diverse cohort. To be eligible, participants must be 40 years or older, not pregnant, and not currently taking antibiotics. At baseline, participants complete demographic questionnaires and (optional) food frequency questionnaires, and provide oral and stool samples. To date, FAMiLI has recruited over 4000 participants, with planned continued recruitment and prospective follow-up for disease outcomes. This study was approved by the NYU Langone Health Institutional Review Board (\#s12-00855), and all participants provided written informed consent.

\section{Participants}

For the current analysis, participants were selected from the FAMiLI parent cohort who had completed demographic and diet questionnaires, and provided both oral and stool samples. In total, 873 were selected for microbiome sequencing. Participants with missing or unknown race or country of origin were excluded from all analyses, with the exception of whites with missing country who were assumed to have been born in the United States $(n=5)$ and Koreans with missing country who were assumed to have been born in Korea $(n=7)$. This exclusion resulted in 863 subjects remaining, including 331 Whites, 97 Blacks, 145 Hispanics (participants identifying as both Hispanic and Black $[n=12]$ were categorized under Hispanic), and 290 Asians. We then created data subsets for each type of analysis: (a) diet alone $(n=816)$, (b) microbiome alone $(n=829)$, and (c) diet and microbiome $(n=785)$, as described below.

\section{Dietary assessment}

Participants completed the 137-item food frequency questionnaire derived from the National Cancer Institute Prostate, Lung, Colorectal, and Ovarian Cancer screening trial, known in the trial as the dietary questionnaire (DQX) [12]. The DQX was translated into Korean and Spanish, respectively, for the Korean- and Spanish-speaking participants. For dietary analysis, we excluded participants with missing diet, with majority of DQX questions or any DQX sections left blank, or with majority of DQX questions answered with multiple responses per food line item. For all other questionnaires, blanks were assumed to be zeroes and the lower frequency response was selected in the case of multiple responses per food line item. After exclusions, there were 816 subjects remaining for dietary analysis.

As the propensity to eat certain foods is pertinent to dietary acculturation, we used food frequencies in our analysis rather than grams or energy adjusted grams. Weekly food frequencies were calculated based on frequency values in Appendix 11 of the PLCO DQX Data Dictionary (National Cancer Institute Cancer Data Access System). Weekly frequencies were multiplied by the number of weeks per year of the food item consumption (based on DQX Appendix 9: 13 weeks for in season fruits/vegetables, 39 weeks for out of season fruits/vegetables, 52 weeks for all other foods), and then divided by 365.25 to arrive at daily food frequencies. Food items were hierarchically categorized into What We Eat in America (WWEIA) food categories, according to food assignments in the USDA Food and Nutrient Database for Dietary Studies (Supplementary Table 1). Food items belonging to multiple categories $(n=5)$ were split evenly into the respective categories. We treated this hierarchical food categorization as a "cladogram" of foods (Supplementary Fig. 1). The generalized UniFrac distance ( $\alpha=0.50)$, based on daily food frequencies and the food "cladogram", was used to characterize diet dissimilarities between participants in the study, similar to the method in Vangay et al. [11]. Diet dissimilarities were visualized using principal coordinate analysis $(\mathrm{PCoA})$.

\section{Microbiome assessment}

Stool samples underwent 16S rRNA gene sequencing at the Environmental Sample Preparation and Sequencing Facility at Argonne National Laboratory. DNA extraction and amplification was performed in six batches, across which the different study race/ethnicity groups were mixed; all samples were pooled for sequencing. DNA was extracted using the Mo Bio PowerSoil DNA isolation kit, following the manufacturer's protocol. The V4 region of the $16 \mathrm{~S}$ rRNA gene was PCR amplified with the 515F/806R primer pair, which included sequencer adapter sequences used in the Illumina flowcell and sample-specific barcodes [13, 14]. Each $25 \mu \mathrm{L}$ PCR reaction contained $9.5 \mu \mathrm{L}$ of Mo Bio PCR Water (Certified DNA-Free), $12.5 \mu \mathrm{L}$ of QuantaBio's AccuStart II PCR ToughMix ( $2 \times$ concentration, $1 \times$ final), $1 \mu \mathrm{L}$ Golay barcode tagged Forward Primer $(5 \mu \mathrm{M}$ concentration, $200 \mathrm{pM}$ final), $1 \mu \mathrm{L}$ Reverse Primer $(5 \mu \mathrm{M}$ concentration, $200 \mathrm{pM}$ final), and $1 \mu \mathrm{L}$ of template DNA. The conditions for PCR were as follows: $94{ }^{\circ} \mathrm{C}$ for $3 \mathrm{~min}$ to 
denature the DNA, with 35 cycles at $94{ }^{\circ} \mathrm{C}$ for $45 \mathrm{~s}, 50{ }^{\circ} \mathrm{C}$ for $60 \mathrm{~s}$, and $72{ }^{\circ} \mathrm{C}$ for $90 \mathrm{~s}$; with a final extension of $10 \mathrm{~min}$ at $72{ }^{\circ} \mathrm{C}$. PCR products were quantified using PicoGreen (Invitrogen) and a plate reader (Infinite 200 PRO, Tecan). Sample PCR products were then pooled in equimolar amounts, purified using AMPure XP Beads (Beckman Coulter), and then quantified using a fluorometer (Qubit, Invitrogen). Molarity was then diluted to $2 \mathrm{nM}$, denatured, and then diluted to a final concentration of $6.75 \mathrm{pM}$ with a $10 \%$ PhiX spike for sequencing on the Illumina MiSeq. Amplicons were sequenced on a $151 \mathrm{bp} \times 12 \mathrm{bp} \times 151 \mathrm{bp}$ MiSeq run [14]. Sequence reads were processed using QIIME 2 [15]. Briefly, sequence reads were demultiplexed and paired-end reads were joined, followed by quality filtering as described in Bokulich et al. [16]. Next the Deblur workflow was applied, which uses sequence error profiles to obtain putative error-free sequences, referred to as "sub" operational taxonomic units (s-OTU) [17]. s-OTUs were assigned taxonomy using a naive Bayes classifier pretrained on the Greengenes [18] 13_8 99\% OTUs, where the sequences have been trimmed to only include 250 bases from the $16 \mathrm{~S}$ V4 region, bound by the $515 \mathrm{~F} / 806 \mathrm{R}$ primer pair. A phylogenetic tree was constructed via sequence alignment with MAFFT [19], filtering the alignment, and applying FastTree [20] to generate the tree. Number of observed s-OTUs and the Shannon Diversity Index were calculated in 100 iterations at 50 different rarefiedsequencing depths (from 15 to 5000 sequence reads per sample), and averaged for each subject at each depth, to generate rarefaction curves (Supplementary Fig. 2).

For microbiome analysis, we excluded subjects for whom sequencing failed $(n=9)$, and subjects with sequencing depths $<250$ sequence reads per sample after the Deblur workflow $(n=25)$, based on visual observation in the rarefaction curves that this depth sufficiently represents the diversity of the samples in this study (Supplementary Fig. 2). After these exclusions, there were 829 subjects remaining for microbiome analysis, with sequencing depths after Deblur ranging from 271 to $25550(\mathrm{Q} 1=3516, \mathrm{Q} 2=$ 6664, Q3 = 8992).

The generalized UniFrac distance and Jensen Shannon Divergence (JSD) were used to characterize dissimilarities in overall microbiome composition between subjects, and dissimilarities were visualized using PCoA.

\section{Statistical analysis}

Permutational multivariate analysis of variance was used to test differences in overall microbiome composition, as measured by the generalized UniFrac distance and JSD, between study groups, adjusting for age and sex (reduced model) or age, sex, smoking, marital status, education, BMI, and exercise (full model). The $W_{\mathrm{d}} *$ test [21], which tests differences in overall microbiome composition while accounting for differences in group dispersion, was also used to ensure there was no confounding by dispersion effects. Distance-based redundancy analysis (db-RDA) implemented in the $\mathrm{R}$ "vegan" package was used to examine the association between diet PCs and overall microbiome composition. The Analysis of Composition of Microbiomes (ANCOM) method [22] was used to detect taxa associated with race/ethnicity groups and with diet, adjusting for age and sex (reduced model) or age, sex, smoking, marital status, education, BMI, and exercise (full model). An ANCOM detection level $\geq 0.6$ was considered significant. Differential abundance of taxa was visualized in cladograms using the $\mathrm{R}$ "metacoder" package [23]. All analyses were conducted in $\mathrm{R}$ (version 3.5.1).

\section{Results}

\section{Study groups}

The study population of 863 US residents comprised 331 Whites, 97 Blacks, 145 Hispanics, and 290 Asians. The majority of White (95\%) and Black (96\%) participants were born in the United States, while the majority of Asian participants were born in Korea (91\%) and the majority of Hispanic participants were born outside the United States (72\%). Foreign-born Hispanic participants were from Puerto Rico (30\%), Mexico (21\%), Dominican Republic (15\%), other Central American countries (14\%), other South American countries (18\%), Cuba (1\%), and Spain (1\%). Because race/ethnic categories were strongly linked with US nativity in this study population, we categorized participants by race/ethnicity and US nativity for all analyses. Demographic characteristics for the five larger groups (US-born Whites, Blacks, and Hispanics, and foreign-born Hispanics and Koreans) are shown in Table 1. The groups differed significantly on all characteristics (age, sex, smoking, BMI, education, marital status, and exercise), as might be expected given different origins, cultures, and lifestyles of these groups. The groups also differed in selfreported disease prevalence (Supplementary Table 2).

\section{Differences in microbiome diversity and composition by race/ethnicity and birthplace}

The study groups did not differ significantly in number of observed s-OTUs (richness) or the Shannon Diversity Index (Kruskal-Wallis $p=0.13$ and $p=0.20$, respectively) (Fig. 1a). However, the groups differed from each other in overall microbiome composition, as measured by the JSD and generalized UniFrac distance (Fig. 1b, c; Supplementary Table 3). The difference between foreign-born Koreans 
Table 1 Demographic characteristics of study participants in the Food and Microbiome Longitudinal Investigation (FAMiLI), by race/ ethnicity and birthplace ${ }^{1}$.

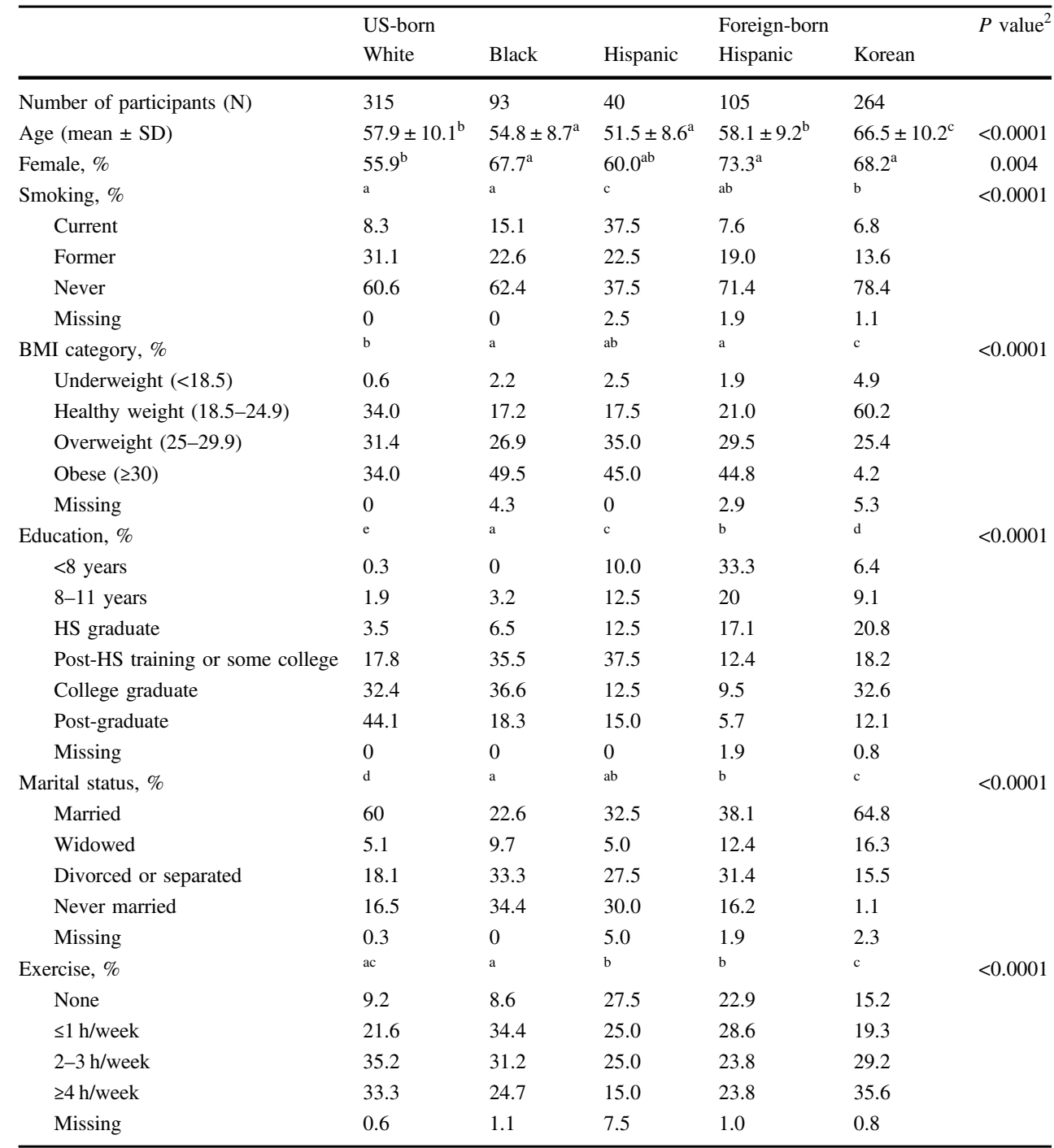

${ }^{1}$ Superscript letters in the table indicate pairwise comparison significance (Wilcoxon rank-sum test for continuous variables and Fisher's exact test for categorical variables). Missing categories were not included for significance testing. Equal letters indicate no significant differences. A significant difference $(p<0.05)$ is indicated when two groups do not have any letters in common.

${ }^{2} P$ values from Kruskal-Wallis test for continuous variables and Fisher's exact test for categorical variables. and US-born Whites explained the most variation in microbiome composition in this study (JSD $R^{2}=2.75 \%$, $p=0.001$; gUniFrac $\left.R^{2}=2.00 \%, p=0.001\right)$, while the difference between US-born Blacks and US-born Hispanics explained the least variation (JSD $R^{2}=0.17 \%, p=0.07$; gUniFrac $R^{2}=0.12 \%, p=0.44$ ), adjusting for age and sex. The amounts of variation explained by group differences were slightly attenuated when further adjusting for potential mediators or confounders (smoking, BMI, marital status, education, and exercise), yet these factors were not fully responsible for group differences (Supplementary Table 3). Foreign-born Koreans were the most homogenous in terms of within-group microbiome composition $(p=0.002$ for difference in dispersion between foreign-born Koreans and
US-born Whites; Fig. 1c). The $W_{\mathrm{d}}{ }^{*}$-test [21] confirmed that group differences in overall microbiome composition were not confounded by differences in group dispersion (Supplementary Table 3). Comparisons by US nativity within the racial/ethnic groups were limited due to small sample sizes for foreign-born Whites, foreign-born Blacks, and USborn Asians (Supplementary Fig. 3); however, we were able to detect a significant difference in overall microbiome composition between US-born and foreign-born Hispanics (JSD $R^{2}=0.38 \%, p=0.001 ; \operatorname{gUniFrac} R^{2}=0.24 \%, p=$ 0.01; Supplementary Table 3 ).

Based on previous literature showing an increase in the Bacteroides/Prevotella ratio upon immigration to the United States [11], we examined whether this ratio differed 

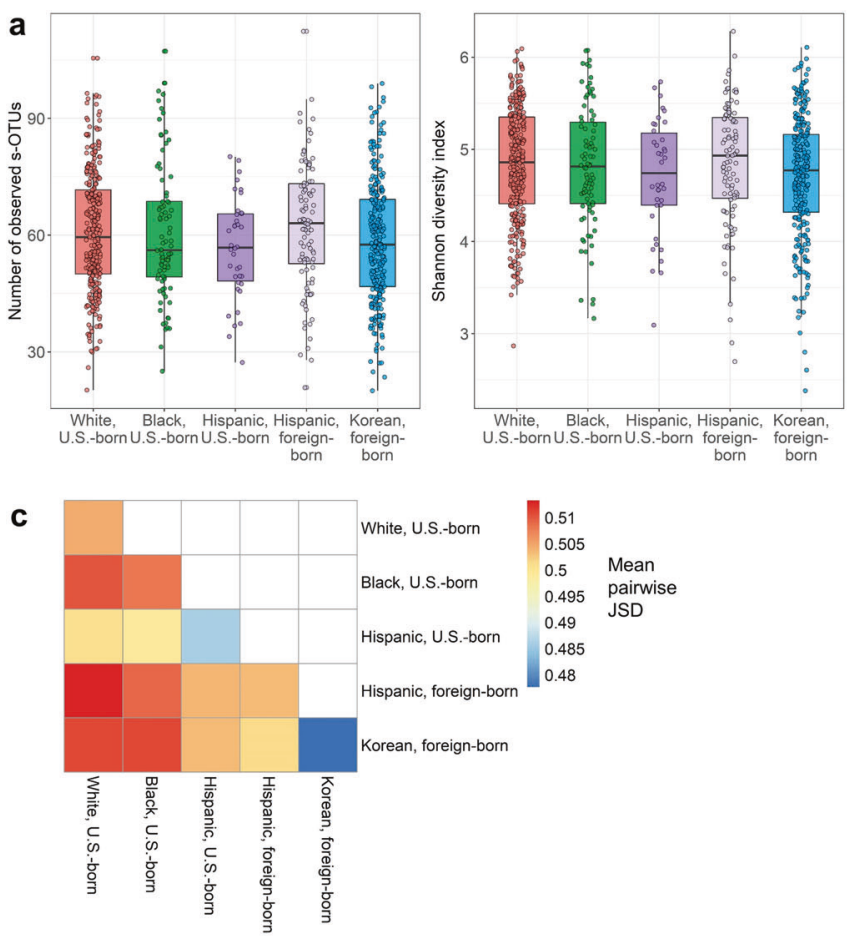

Fig. 1 Gut microbiome $\alpha$ - and $\beta$-diversity according to race/ethnicity and birthplace. a Boxplots of the number of observed s-OTUs and Shannon diversity index by study group. b Principal coordinate analysis of the Jensen Shannon Divergence (JSD). c Mean pairwise

across our study groups. We observed that the Bacteroides/ Prevotella ratio was significantly higher in US-born Whites, compared with US-born Blacks, foreign-born Hispanics, and foreign-born Koreans $(p=0.02,0.0002$, and 0.0002 from the Dunn's test; Fig. 2a). Using ANCOM, we also identified s-OTUs and higher level taxa that were differentially abundant between the study groups, adjusting for age and sex (Supplementary Table 4) or age, sex, smoking, BMI, marital status, education, and exercise (Supplementary Table 5). Results were highly similar between the reduced and fully adjusted models. The most differentially abundant s-OTUs were identified for foreign-born Koreans vs. US-born Whites, followed by foreign-born Hispanics vs. US-born Whites (Fig. 2b). s-OTUs from Prevotella copri and Dialister (enriched in foreign-born Koreans), and Oscillospira, Bacteroides uniformis, and [Ruminococcus] torques (enriched in US-born Whites), were most strongly related to dissimilarities in gut microbiome composition (Supplementary Table 6 and Supplementary Fig. 4). Clustering of differential abundance effects showed that differences in s-OTU abundance between foreign-born and USborn groups tended to be distinct from differences between US-born groups (Fig. 2c).

Comparisons at higher taxonomic levels also revealed many differences between groups (Fig. 2d, e; Supplementary Fig. 5; Supplementary Tables 4 and 5), including
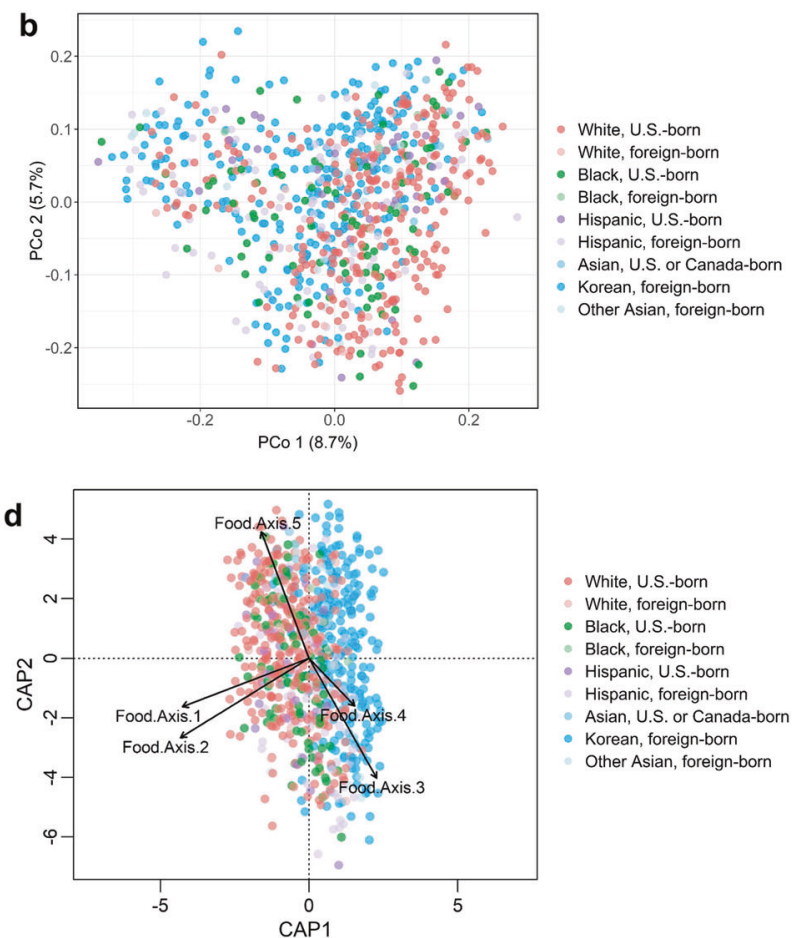

JSD distances between and within study groups. d distance-based redundancy analysis of the JSD, constrained by the first five principal coordinates of the generalized UniFrac diet distance.

elevated abundance of P. copri and Bifidobacterium adolescentis in foreign-born Korean and foreign-born Hispanic participants compared with US-born White participants.

\section{Measurement of dietary acculturation}

Diet dissimilarities between participants were calculated using the generalized UniFrac distance (see Dietary assessment section, "Materials and methods"). Unsupervised hierarchical clustering and principal coordinate (PC) analysis of the diet distances (Fig. 3a, b) revealed particularly strong separation of dietary patterns for USborn White and foreign-born Korean participants. US-born Black and US-born Hispanic diets tended to overlap with US-born White diets on the PC plot (Fig. 3b). In general, PC1 was negatively correlated with intake of vegetables and fruits, and positively correlated with intake of processed meats, refined grains (e.g. white bread), snacks and sweets, dairy products, certain mixed dishes (e.g. pizza, macaroni, and cheese), fats and oils, and coffee and tea (Supplementary Fig. 6). PC2 had similar directions of association with foods as PC1, except that it was negatively correlated with coffee and tea. Based on the angle of the centroids of the US-born White group and the foreign-born Korean group, we rotated the plot to obtain a new rotated axis achieving maximal separation between these two groups (Fig. 3c). 


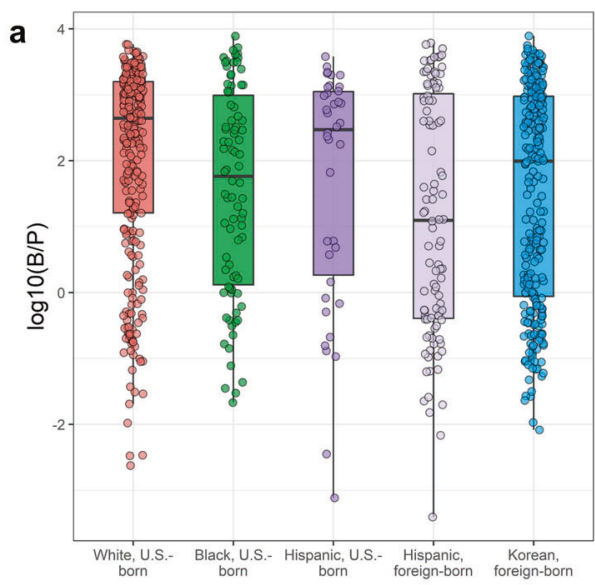

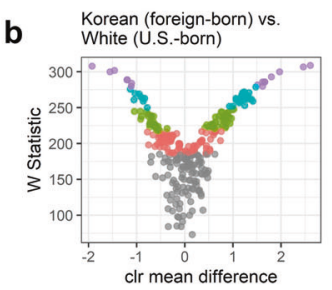
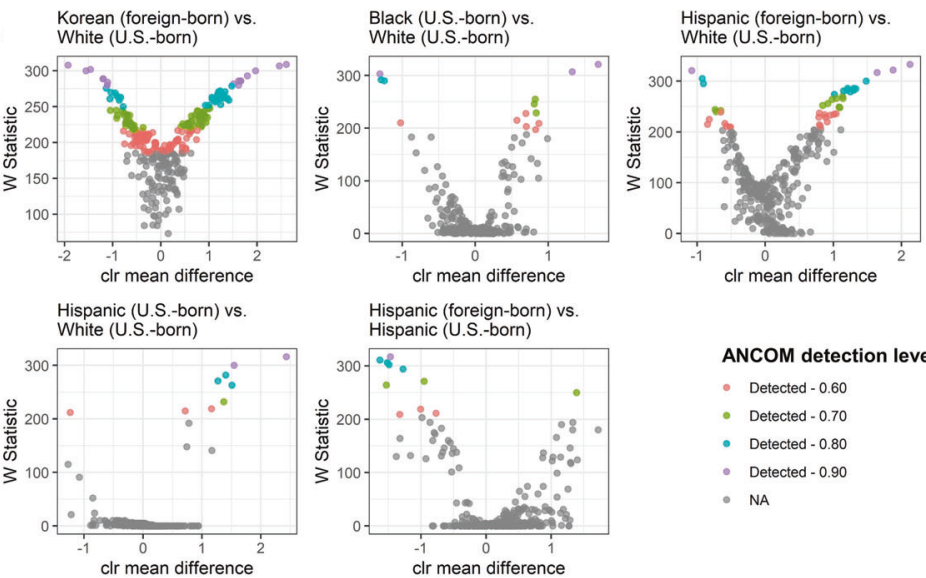

ANCOM detection leve

- Detected -0.60

- Detected -0.70

- Detected -0.80

- Detected -0.90

- NA

c

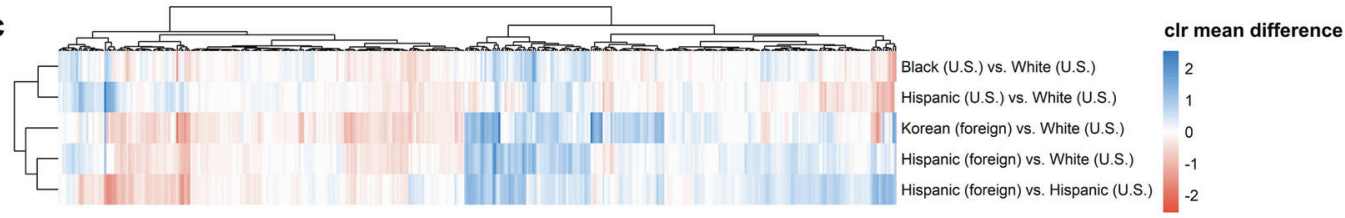

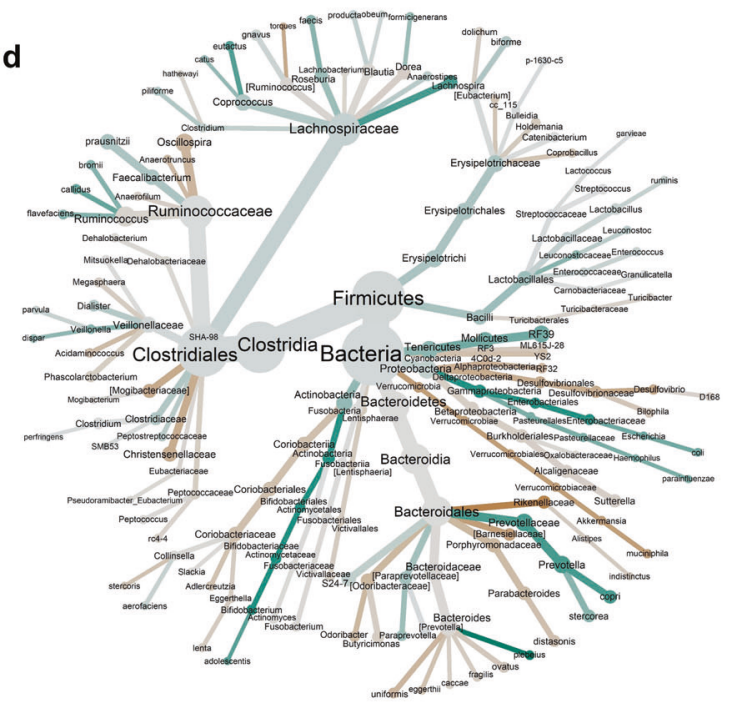

Fig. 2 Differentially abundant taxa by race/ethnicity and birthplace. a Boxplots of the log Bacteroides/Prevotella ratio. b Volcano plots showing differentially abundant s-OTUs as detected by ANCOM (age and sex adjusted model). The $x$-axis represents the difference in mean centered $\log$ ratio (clr)-transformed abundance between groups, and the $y$-axis represents the ANCOM W Statistic. s-OTU points are colored by level of ANCOM significance, with 0.9 being the highest

This new representation was used as the measure of dietary acculturation, with more positive on the axis being more diet acculturated (i.e., more similar to US-born White diet), and more negative on the axis being less diet acculturated (i.e., further from US-born White diet). The dietary acculturation index was largely not associated with demographic or disease characteristics within the study groups, with a few exceptions. In US-born Whites, the index was higher in current smokers and in individuals who did not exercise; in US-born Hispanics, the index was higher in individuals

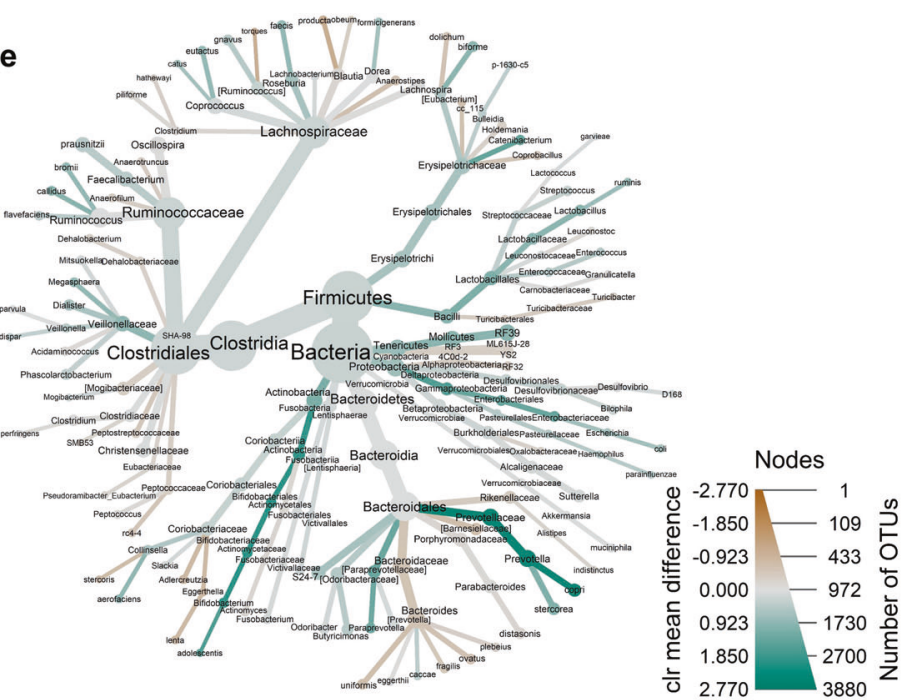

level; s-OTUs in gray were not significant. c Heatmap of clr mean differences of s-OTUs for each study group comparison. d, e Cladograms of phylum through species level taxa; color represents clr mean difference between foreign-born Korean and US-born White participants (d) or foreign-born Hispanic and US-born White participants (e); size of node represents number of s-OTU members; only taxa tested in ANCOM are displayed.

with colorectal polyps; in foreign-born Hispanics, the index was higher in men than in women; and in foreign-born Koreans, the index was lower in individuals with hypertension (Supplementary Table 7).

\section{Dietary acculturation and the gut microbiome}

The first five diet PC (accounting for $36 \%$ of dietary variation) explained $2.7 \%$ of variation in microbiome composition $(p=0.001)$ in distance-based redundancy analysis of 

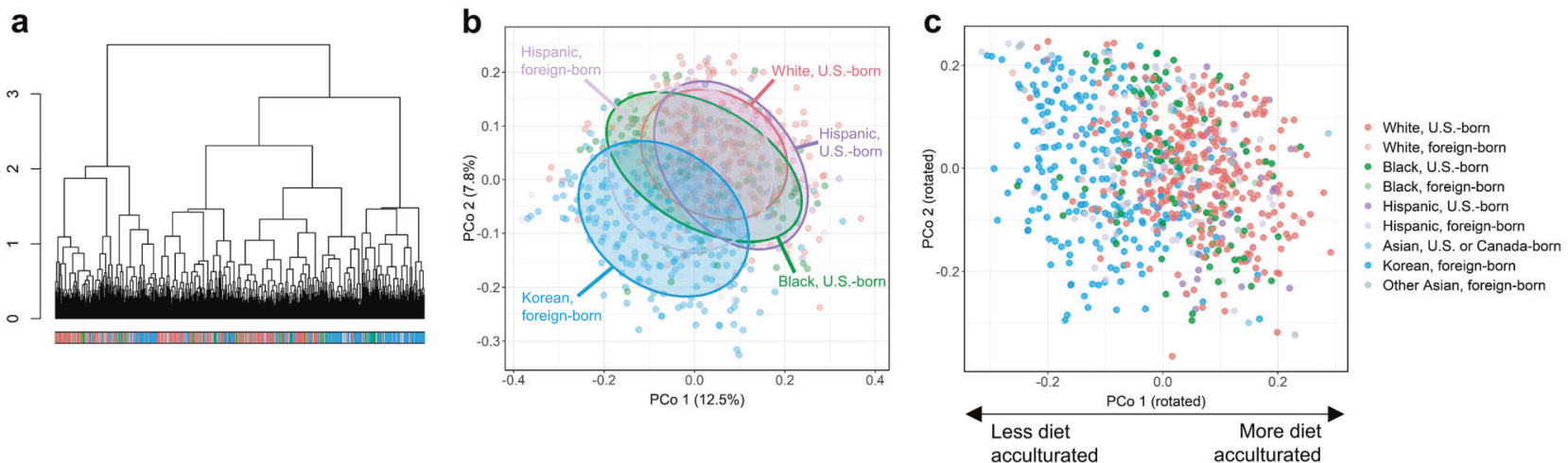

Fig. 3 Measuring dietary acculturation. a Unsupervised hierarchical clustering (Ward's method) of the generalized UniFrac diet distance. b Principal coordinate analysis of the generalized UniFrac diet distance, with $75 \%$ data ellipses overlayed on the plot. c $46.8^{\circ}$ rotation of the plot in (b), corresponding to the angle of the centroids for the
Korean, foreign-born and White, US-born groups. The new rotated first axis, a measure of dietary acculturation, achieves high separation of diet patterns between the Korean, foreign-born and White, US-born groups. the JSD. The first two diet axes were most related to separation in microbiome composition between foreignborn Korean and US-born White participants (Fig. 1d). After controlling the effect of race/ethnicity and birthplace, diet remained significantly associated with microbiome composition $(1.1 \%, p=0.01)$. Similarly, the measure of dietary acculturation also explained a significant proportion of variation in microbiome composition in this study population $(1.5 \%, p=0.001)$, even after controlling for race/ethnicity and birthplace $(0.3 \%, p=0.02)$. Results were similar when additionally controlling for age and sex. Higher dietary acculturation was associated with reduced gut microbiome diversity in both foreign-born Korean and Hispanic participants, though not reaching significance in either group ( $p=0.08$ and 0.11 , respectively; Fig. 4a, b).

In ANCOM analysis, we identified s-OTUs that were associated with the dietary acculturation index in foreignborn Korean participants (Fig. 4c) and foreign-born Hispanic participants (Fig. 4d). In foreign-born Korean participants, s-OTUs from Bacteroides plebeius and Erysipelotrichaceae were depleted with increasing dietary acculturation, while an s-OTU from Bilophila was enriched with increasing dietary acculturation. These s-OTUs were weakly correlated with individual food intake frequencies in foreign-born Koreans (Supplementary Fig. 7). The Bilophila s-OTU had weak positive associations with some processed meats (lunch meat and hot dogs), some sweets (cake, pie, doughnuts, ice cream, and candy), cottage cheese, and some condiments (salad dressing, margarine, sour cream, sweet cream, and butter); the Erysipelotrichaceae s-OTU was negatively associated with some snacks and sweets (pie, doughnuts, cookies, ice cream, candy, chocolate, and salty snacks); and the B. plebeius sOTU was positively associated with brown or wild rice, and negatively associated with some processed meat (cured ham, hot dogs, and bacon), fried fish, fried chicken, cold cereal, cake, crackers, cheeses, butter, and gravies made with meat drippings.

In foreign-born Hispanic participants, two s-OTUs from Ruminococcaceae (one from the Oscillospira genus and one of unknown genus) were depleted with increasing dietary acculturation, and an s-OTU from Erysipelotrichaceae was enriched with increasing dietary acculturation (Fig. 4d). Note that it was not the same s-OTU from Erysipelotrichaceae that was associated with dietary acculturation in the foreign-born Korean and foreign-born Hispanic groups. These s-OTUs were strongly correlated with some individual food intake frequencies in foreign-born Hispanics (Supplementary Fig. 7). For example, the Ruminococcaceae (unknown genus) s-OTU had a strong negative association with eggs, the Oscillospira s-OTU was negatively associated with pork chops and steaks, and the Erysipelotrichaceae s-OTU was positively associated with liver.

\section{Dietary acculturation, acculturative microbiota, and BMI}

As duration of US residence is related to increased risk of obesity in immigrants [5], we explored whether dietary acculturation may contribute to increases in BMI. The dietary acculturation index was not correlated with BMI in the foreign-born Korean participants or the foreign-born Hispanic participants, and the dietary acculturation-related s-OTUs were also not correlated with BMI in these study groups (all $p>0.05$; Supplementary Fig. 8a, b). In US-born Whites, there was a weak positive correlation between the dietary acculturation index and BMI $(R=0.12, p=0.04)$, and two s-OTUs that were depleted with increasing dietary acculturation (one in foreign-born Korean and one in foreign-born Hispanic participants) were inversely 

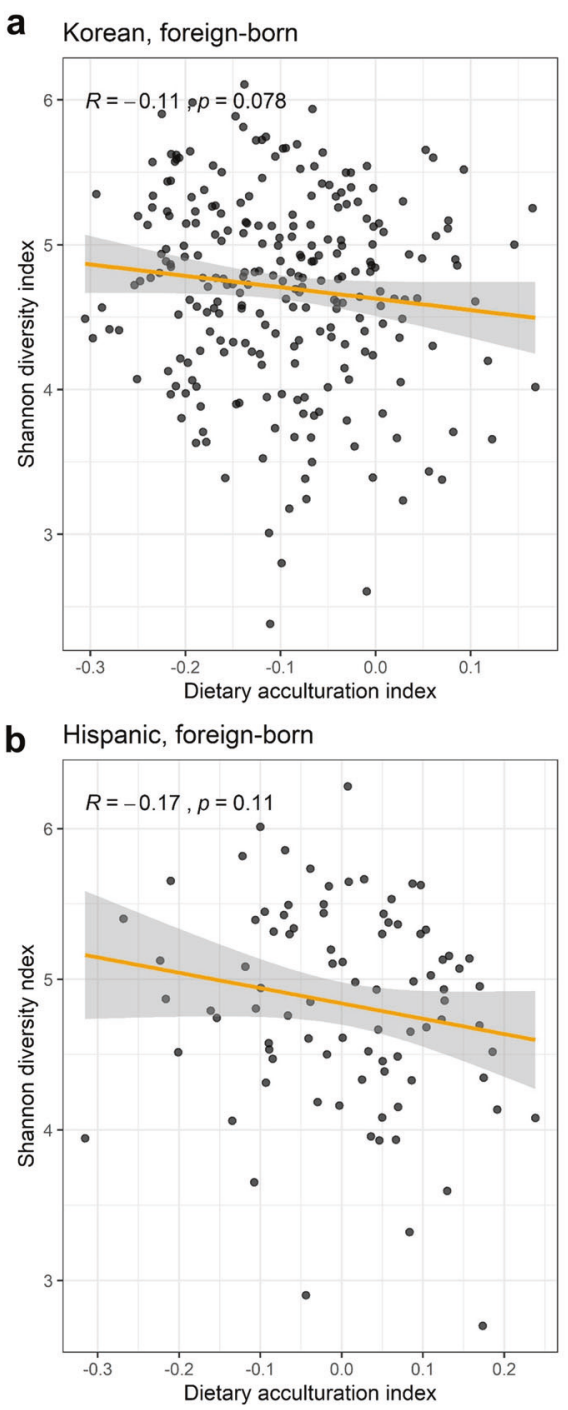

Fig. 4 Dietary acculturation and the gut microbiome in foreignborn Korean and Hispanic participants. a, b Scatterplots of the dietary acculturation index vs. the Shannon diversity index in Korean, foreign-born participants $(R=-0.11, p=0.08)$ and Hispanic, foreignborn participants $(R=-0.17, p=0.11)$. c, d Boxplots of clrtransformed abundance for s-OTUs significantly associated with the dietary acculturation index in foreign-born Koreans or foreign-born

associated with BMI in US-born White participants (Supplementary Fig. 8c).

\section{Discussion}

In this first study of the gut microbiome across understudied immigrant and US-born racial and ethnic groups, we observed significant differences in microbiome composition between foreign-born and US-born groups, as well as between US-born groups of differing racial/ethnic backgrounds. Among the comparisons studied, the gut
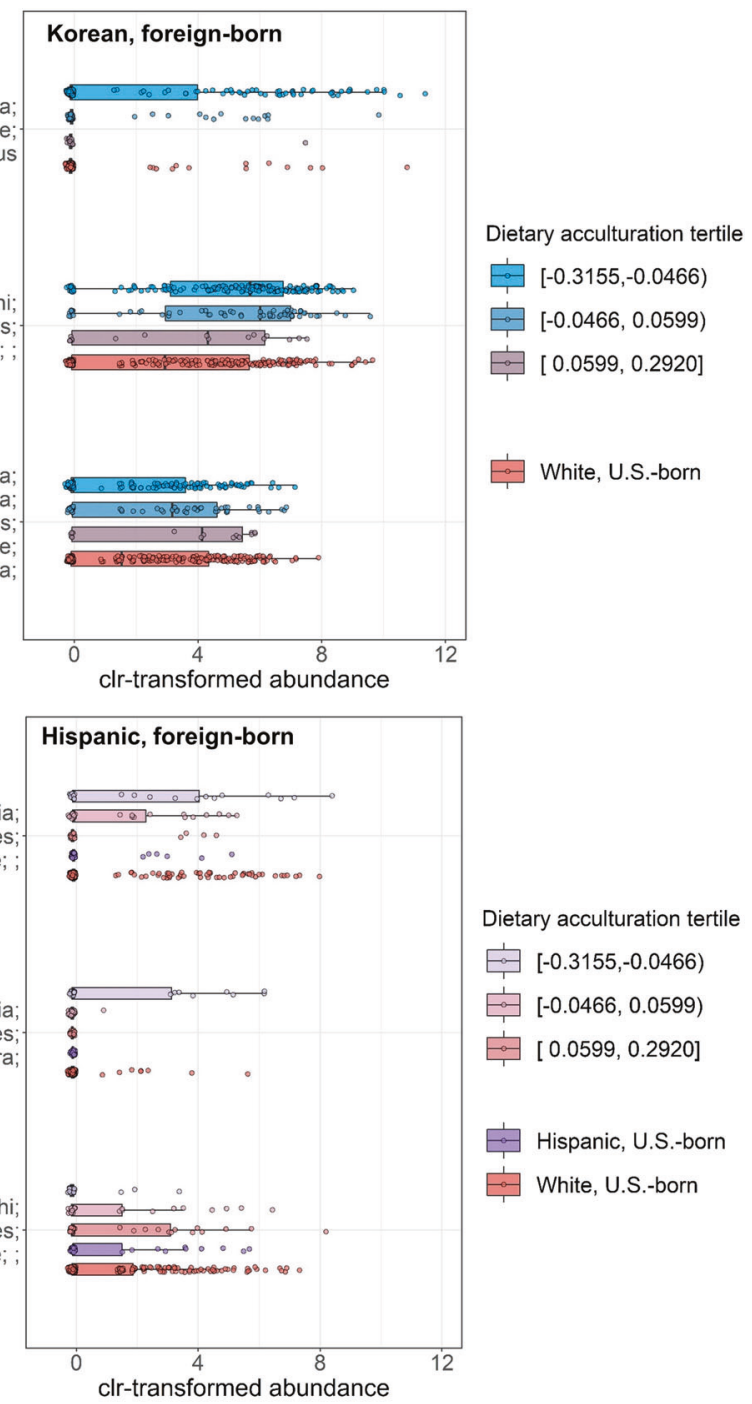

Hispanics in ANCOM analysis (age and sex adjusted model). Tertiles of dietary acculturation were determined among the entire population ( $n=174,59$, and 16 in tertiles 1, 2, and 3 for foreign-born Koreans; $n=28,30$, and 30 in tertiles 1,2 , and 3 for foreign-born Hispanics). Boxplots of clr-transformed abundance in US-born White and USborn Hispanic (plot $\mathbf{d}$ only) participants are included for comparison purposes.

microbiome of foreign-born Korean participants differed most profoundly from US-born White participants. In addition, we used a novel data-driven approach to characterize dietary acculturation in foreign-born Korean and Hispanic participants, and found that dietary acculturation may contribute to acculturation of the gut microbiome. These results highlight the important influence of place of birth and dietary acculturation on the gut microbiome, which may have consequences for immigrant health.

The composition of the human gut microbiome is shaped significantly by extrinsic factors such as diet, lifestyle, and geographic environment $[8,10,24-30]$, all of which can 
change drastically during the acculturation process. Yet little is known regarding the impact of immigration and acculturation on the human gut microbiome. A recent report on Hmong and Karen individuals living in Thailand and the United States found that US immigration results in loss of gut microbiome diversity, displacement of native Prevotella strains with Bacteroides strains, and loss of bacterial enzymes associated with plant fiber degradation [11]. While we did not observe differences in microbiome diversity between study groups, we did observe a decreased ratio of Bacteroides to Prevotella abundance in foreign-born Korean and Hispanic participants compared with US-born Whites, in agreement with the aforementioned study. Higher abundance of $P$. copri in foreign-born Korean and Hispanic participants appeared responsible for this finding. We observed many taxa that differed in abundance between foreign-born Korean and Hispanic participants and US-born Whites, with some overlapping differences (e.g. enrichment of Bifidobacterium, Paraprevotella, and Prevotella in foreign-born groups) and some differences unique to each immigrant group (e.g., enrichment of B. plebeius and depletion of Rikenellaceae in foreign-born Koreans, and enrichment of Lactobacillus and Catenibacterium in foreign-born Hispanics). In addition, we found that s-OTU level patterns were similar when comparing foreign-born Hispanic participants to either US-born Whites or US-born Hispanics, suggesting that place of birth rather than race/ ethnicity is a stronger microbiome determinant and that microbiome acculturation may occur within one or more generations of residence in the United States.

We also observed significant differences in gut microbiome composition between US-born groups, albeit less profound than differences between foreign-born and USborn groups. Interestingly, some of the differences between US-born Blacks and Whites paralleled those of the foreignborn groups vs. US-born White comparison, such as enrichment of Bifidobacterium and Prevotella in US-born Blacks compared with US-born Whites. Though generally, similar patterns were observed at the s-OTU level for USborn Black and US-born Hispanic participants compared with US-born Whites, and these patterns were distinct from that of the foreign-born groups vs. US-born White comparison.

While we did not have shotgun metagenomic data to determine functional consequences of the observed microbiome differences, known functions of some of the identified taxa may provide clues of their roles. Bifidobacterium species are commonly used as food probiotics due to their beneficial roles in human health [31], including their digestive activity toward lactose. Our observation of higher abundance of Bifidobacterium in foreign-born Korean, foreign-born Hispanic, and US-born Black participants compared with US-born Whites may relate to the higher prevalence of lactose intolerance in these populations [32], as individuals with genetic predisposition to lactose intolerance and sufficient dairy consumption have high abundance of Bifidobacterium [33]. However, the lack of genetic information precludes us from drawing this conclusion. Prevotella abundance tends to be high in non-Western immigrants to Westernized countries [11, 34], and appears to decline with increased duration of residence[11]; this is consistent with our finding of higher $P$. copri in foreignborn Koreans and Hispanics compared with US-born Whites, though does not fit with our observation of higher $P$. copri in US-born Blacks. There is substantial strain-level diversity in $P$. copri, particularly related to diet and Western or non-Western origin [35], making it plausible that different $P$. copri strains are prevalent in each of our study groups. Whole metagenome sequencing will be necessary to achieve strain-level resolution in P. copri. Lastly, B. plebeius is known to digest complex carbohydrates in seaweed, and likely acquired this ability via horizontal gene transfer from a marine bacterium many centuries ago [36]. Thus it is not surprising that foreign-born Korean participants in our study were the only group with enrichment of B. plebeius, being the only group under study with seaweed as a regular dietary component.

Dietary acculturation is likely a key factor in shaping the gut microbiome of immigrant populations, as changes in diet can rapidly alter the gut microbiome [24]. In crosssectional population-based studies, diet typically explains a small (i.e., low single digit percentage) but significant proportion of overall microbiome composition when adjusting for other covariates [25, 30], similar to our findings here. In the study on Hmong and Karen individuals in Thailand and the United States, the first five PCs of diet explained $3.2 \%$ of total microbiome variation [11]; similarly in our analysis, we observed that the first five PCs of diet explained $2.7 \%$ of total microbiome variation. Here, we went a step further and used a novel data-driven approach to characterize dietary acculturation, creating a dietary acculturation index which maximized the diet dissimilarity between foreign-born Korean and US-born White participants. Dietary acculturation, as measured by our data-driven index, was marginally associated with reduced gut microbiome diversity in foreign-born Korean and Hispanic participants, and was also associated with abundance of specific s-OTUs in each foreign-born group. Abundance of these s-OTUs in more diet acculturated foreign-born participants resembled abundance in US-born Whites, suggesting that dietary acculturation results in some level of gut microbiome acculturation. Interestingly, dietary acculturation was related to depletion of the seaweed-digesting species B. plebeius in foreign-born Korean participants, indicating that we captured true acculturative changes in diet with this data-driven dietary acculturation index, and 
that dietary acculturation may result in loss of native bacterial strains in immigrant populations. The acculturationrelated s-OTUs were associated with specific food items, including some negative associations between Western diet foods and acculturation-depleted s-OTUs; but observational study results cannot reveal whether microbiome changes are due to increased consumption of Western diet foods, or decreased consumption of native foods. An s-OTU from Bilophila, enriched with higher dietary acculturation in foreign-born Koreans, was related to dietary intake of highfat condiments (e.g. butter, cream), in agreement with research in mice reporting that a high-fat diet promotes expansion of sulfite-reducing Bilophila wadsworthia [37].

The health consequences of diet acculturation-related gut microbiome changes are currently unclear. Increased duration of residence in the United States has been consistently related to increased BMI in both Korean Americans [38, 39] and Hispanic Americans [40], with dietary acculturation possibly playing a role in this relationship [39, 41, 42] along with other factors. In our study, the dietary acculturation index was not associated with BMI in foreign-born Korean or Hispanic participants nor were abundances of diet acculturation-related s-OTUs. However, we lacked information on years of residence in the United States and age at immigration, key variables that may modify the effect of dietary acculturation and acculturation-related microbiome changes on BMI. In US-born White participants, who are higher on the dietary acculturation scale and have presumably followed their reported diets much longer than the foreign-born groups, the dietary acculturation index and diet acculturation-related s-OTUs were significantly related to BMI. This suggests that diet acculturation-related changes in the gut microbiome may impact weight gain over the long term. Further research is necessary to determine the long-term health consequences of changes in the gut microbiome due to dietary acculturation.

Our study was strengthened first and foremost by the diversity of the study population, allowing us to characterize the gut microbiome of understudied populations in the United States. In addition, parallel collection of food frequency data across the diverse study groups allowed us to characterize participant diet and dietary acculturation using a data-driven approach, as opposed to more subjective surveys on food preference (e.g., preference for American or native culture foods) used to characterize dietary acculturation previously [41, 42]. Our study was limited by several factors, including lack of data on duration of residence in the United States, age at arrival, and other acculturation measures, which would allow us to better characterize the effects of immigration and acculturation on the gut microbiome, and lack of shotgun metagenomic data to characterize the functional capacities of the affected microbiota. As this was a cross-sectional study, we were not able to examine within-person changes in diet, microbiome, and other lifestyle factors pre- to post-immigration, as well as the time scale of microbiome changes and the long-term health consequences of dietary acculturation and diet acculturation-related changes in the gut microbiome. Recruitment of the majority of our White and Black participants from an online platform may impact generalizability of findings to the wider population. Smaller sample sizes for the Black and Hispanic participant groups limited power for drawing conclusions in these groups; particularly, the dietary acculturation index was developed based on KoreanWhite differences, limiting its generalizability to the Hispanic population. Finally, we lacked a US-born Korean group, which would be ideal for comparison with the foreign-born Korean group, though other evidence suggests that second generation immigrant microbiomes resemble that of US-born Whites [11].

In conclusion, we found that the place of birth was a significant determinant of the human gut microbiome in a diverse study population of foreign-born and US-born Americans. We developed a measure of dietary acculturation that was significantly related to gut microbiome composition, with particular s-OTU abundance in more dietacculturated foreign-born participants resembling abundance in US-born White participants. These results suggest that dietary acculturation leads to acculturation of the gut microbiome. Further research is needed to determine the health consequences of acculturative changes in the gut microbiota in immigrant populations.

\section{Data availability}

The 16S rRNA sequencing data that support the findings of this study have been deposited in the Sequence Read Archive (PRJNA559143), along with demographic metadata, to be released upon publication. Additional data on the study participants are available from the corresponding author upon reasonable request.

Acknowledgements We thank our partners at Korean Community Services for facilitating recruitment in the Korean community. We are grateful to the FAMiLI participants for contributing their time to participate in this study. This work was supported in part by the NYU Center for the Study of Asian American Health under the NIH National Institute on Minority Health \& Health Disparities grant award \#U54MD000538-15.

\section{Compliance with ethical standards}

Conflict of interest The authors declare that they have no conflict of interest. 
Publisher's note Springer Nature remains neutral with regard to jurisdictional claims in published maps and institutional affiliations.

\section{References}

1. United Nations. International Migration Report 2017; United Nations; New York, 2017.

2. Argeseanu Cunningham S, Ruben JD, Narayan KM. Health of foreign-born people in the United States: a review. Health Place. 2008;14:623-35.

3. Ahmed AT, Quinn VP, Caan B, Sternfeld B, Haque R, Van Den Eeden SK. Generational status and duration of residence predict diabetes prevalence among Latinos: the California Men's Health Study. BMC Public Health. 2009;9:392.

4. Commodore-Mensah Y, Ukonu N, Obisesan O, Aboagye JK, Agyemang C, Reilly CM, et al. Length of residence in the United States is associated with a higher prevalence of cardiometabolic risk factors in immigrants: a contemporary analysis of the National Health Interview Survey. J Am Heart Assoc. 2016;5:e004059.

5. Goel MS, McCarthy EP, Phillips RS, Wee CC. Obesity among US immigrant subgroups by duration of residence. JAMA. 2004;292: 2860-7.

6. Le-Scherban F, Albrecht SS, Bertoni A, Kandula N, Mehta N, Diez Roux AV. Immigrant status and cardiovascular risk over time: results from the Multi-Ethnic Study of Atherosclerosis. Ann Epidemiol. 2016;26:429-35.e421.

7. Schwartz SJ, Unger JB, Zamboanga BL, Szapocznik J. Rethinking the concept of acculturation: implications for theory and research. Am Psychol. 2010;65:237-51.

8. De Filippo C, Cavalieri D, Di Paola M, Ramazzotti M, Poullet JB, Massart S, et al. Impact of diet in shaping gut microbiota revealed by a comparative study in children from Europe and rural Africa. Proc Natl Acad Sci USA. 2010;107:14691-6.

9. Jha AR, Davenport ER, Gautam Y, Bhandari D, Tandukar S, Ng $\mathrm{KM}$, et al. Gut microbiome transition across a lifestyle gradient in Himalaya. PLoS Biol. 2018;16:e2005396.

10. Yatsunenko T, Rey FE, Manary MJ, Trehan I, Dominguez-Bello MG, Contreras M, et al. Human gut microbiome viewed across age and geography. Nature. 2012;486:222-7.

11. Vangay P, Johnson AJ, Ward TL, Al-Ghalith GA, Shields-Cutler RR, Hillmann BM, et al. US immigration westernizes the human gut microbiome. Cell. 2018;175:962-72.e910.

12. National Cancer Institute Cancer Data Access System. Dietary Questionnaire (DQX) Datasets. https://cdas.cancer.gov/datasets/ $\mathrm{plco} / 97 /$.

13. Caporaso JG, Lauber CL, Walters WA, Berg-Lyons D, Lozupone CA, Turnbaugh PJ, et al. Global patterns of $16 \mathrm{~S}$ rRNA diversity at a depth of millions of sequences per sample. Proc Natl Acad Sci USA. 2011;108 Suppl 1:4516-22.

14. Caporaso JG, Lauber CL, Walters WA, Berg-Lyons D, Huntley J, Fierer N, et al. Ultra-high-throughput microbial community analysis on the Illumina HiSeq and MiSeq platforms. ISME J. 2012;6:1621-4.

15. Caporaso JG, Kuczynski J, Stombaugh J, Bittinger K, Bushman FD, Costello EK, et al. QIIME allows analysis of high-throughput community sequencing data. Nat Methods. 2010;7:335-6.

16. Bokulich NA, Subramanian S, Faith JJ, Gevers D, Gordon JI, Knight R, et al. Quality-filtering vastly improves diversity estimates from Illumina amplicon sequencing. Nat Methods. 2013;10:57-59.

17. Amir A, McDonald D, Navas-Molina JA, Kopylova E, Morton JT, Zech Xu Z, et al. Deblur rapidly resolves single-nucleotide community sequence patterns. mSystems. 2017;2:e00191-16.

18. DeSantis TZ, Hugenholtz P, Larsen N, Rojas M, Brodie EL, Keller K, et al. Greengenes, a chimera-checked 16S rRNA gene database and workbench compatible with ARB. Appl Environ Microbiol. 2006;72:5069-72.

19. Katoh K, Misawa K, Kuma K, Miyata T. MAFFT: a novel method for rapid multiple sequence alignment based on fast Fourier transform. Nucleic Acids Res. 2002;30:3059-66.

20. Price MN, Dehal PS, Arkin AP. FastTree: computing large minimum evolution trees with profiles instead of a distance matrix. Mol Biol Evol. 2009;26:1641-50.

21. Hamidi B, Wallace K, Vasu C, Alekseyenko AV. W(*)d -test: robust distance-based multivariate analysis of variance. Microbiome. 2019;7:51

22. Mandal S, Van Treuren W, White RA, Eggesbo M, Knight R, Peddada SD. Analysis of composition of microbiomes: a novel method for studying microbial composition. Microb Ecol Health Dis. 2015;26:27663.

23. Foster ZSL, Sharpton TJ, Grünwald NJ. Metacoder: an R package for visualization and manipulation of community taxonomic diversity data. PLoS Comput Biol. 2017;13:e1005404.

24. David LA, Maurice CF, Carmody RN, Gootenberg DB, Button JE, Wolfe BE, et al. Diet rapidly and reproducibly alters the human gut microbiome. Nature. 2014;505:559-63.

25. Falony G, Joossens M, Vieira-Silva S, Wang J, Darzi Y, Faust K, et al. Population-level analysis of gut microbiome variation. Science. 2016;352:560-4.

26. Rothschild D, Weissbrod O, Barkan E, Kurilshikov A, Korem T, Zeevi D, et al. Environment dominates over host genetics in shaping human gut microbiota. Nature. 2018;555:210.

27. Turnbaugh PJ, Ridaura VK, Faith JJ, Rey FE, Knight R, Gordon JI. The effect of diet on the human gut microbiome: a metagenomic analysis in humanized gnotobiotic mice. Sci Transl Med. 2009;1:6ra14.

28. Wu GD, Chen J, Hoffmann C, Bittinger K, Chen YY, Keilbaugh $\mathrm{SA}$, et al. Linking long-term dietary patterns with gut microbial enterotypes. Science. 2011;334:105-8.

29. Xu Z, Knight R. Dietary effects on human gut microbiome diversity. Br J Nutr. 2015;113 Suppl:S1-5.

30. Zhernakova A, Kurilshikov A, Bonder MJ, Tigchelaar EF, Schirmer M, Vatanen T, et al. Population-based metagenomics analysis reveals markers for gut microbiome composition and diversity. Science. 2016;352:565-9.

31. Lee J-H, O'Sullivan DJ. Genomic insights into bifidobacteria. Microbiol Mol Biol Rev. 2010;74:378-416.

32. Swagerty DL Jr., Walling AD, Klein RM. Lactose intolerance. Am Fam Physician. 2002;65:1845-50.

33. Bonder MJ, Kurilshikov A, Tigchelaar EF, Mujagic Z, Imhann F, Vila AV, et al. The effect of host genetics on the gut microbiome. Nat Genet. 2016;48:1407.

34. Deschasaux M, Bouter KE, Prodan A, Levin E, Groen AK, Herrema $\mathrm{H}$, et al. Depicting the composition of gut microbiota in a population with varied ethnic origins but shared geography. Nat Med. 2018;24:1526-31.

35. De Filippis F, Pasolli E, Tett A, Tarallo S, Naccarati A, De Angelis $M$, et al. Distinct genetic and functional traits of human intestinal prevotella copri strains are associated with different habitual diets. Cell Host Microbe. 2019;25: 444-53.e443.

36. Hehemann J-H, Correc G, Barbeyron T, Helbert W, Czjzek M, Michel G. Transfer of carbohydrate-active enzymes from marine bacteria to Japanese gut microbiota. Nature. 2010;464:908.

37. Devkota S, Wang Y, Musch MW, Leone V, Fehlner-Peach H, Nadimpalli A, et al. Dietary-fat-induced taurocholic acid promotes pathobiont expansion and colitis in Il10-/- mice. Nature. 2012; 487:104-8.

38. Cho J, Juon H-S. Assessing overweight and obesity risk among Korean Americans in California using World Health Organization 
body mass index criteria for Asians. Prev Chronic Dis. 2006; 3:A79.

39. Chen L, Juon HS, Lee S. Acculturation and BMI among Chinese, Korean and Vietnamese adults. J Community Health. 2012;37:539-46.

40. Park J, Myers D, Kao D, Min S. Immigrant obesity and unhealthy assimilation: alternative estimates of convergence or divergence, 1995-2005. Soc Sci Med. 2009;69:1625-33.
41. Isasi CR, Ayala GX, Sotres-Alvarez DT, Madanat H, Penedo F, Loria CM, et al. Is acculturation related to obesity in Hispanic/ Latino Adults? Results from the Hispanic Community Health Study/Study of Latinos. J Obes. 2015;2015:186276.

42. Song YJ, Hofstetter CR, Hovell MF, Paik HY, Park HR, Lee J, et al. Acculturation and health risk behaviors among Californians of Korean descent. Prev Med. 2004;39:147-56. 